\title{
The JWST/NIRCam coronagraph flight occulters
}

John E. Krist, Kunjithapatham Balasubramanian, Richard E. Muller, Stuart B. Shaklan, Douglas M. Kelly, et al.

John E. Krist, Kunjithapatham Balasubramanian, Richard E. Muller, Stuart B. Shaklan, Douglas M. Kelly, Daniel W. Wilson, Charles A. Beichman, Eugene Serabyn, Yalan Mao, Pierre M. Echternach, John T. Trauger, Kurt M. Liewer, "The JWST/NIRCam coronagraph flight occulters," Proc. SPIE 7731, Space Telescopes and Instrumentation 2010: Optical, Infrared, and Millimeter Wave, 77313J (10 August 2010); doi: 10.1117/12.856488

SPIE Event: SPIE Astronomical Telescopes + Instrumentation, 2010, San Diego, California, United States 


\title{
The JWST/NIRCam coronagraph flight occulters
}

\author{
John E. Krist ${ }^{\mathrm{a}}$, Kunjithapatham Balasubramanian ${ }^{\mathrm{a}}$, Richard E. Muller ${ }^{\mathrm{a}}$, Stuart B. Shaklan ${ }^{\mathrm{a}}$, \\ Douglas M. Kelly ${ }^{\mathrm{b}}$, Daniel W. Wilson ${ }^{\mathrm{a}}$, Charles A. Beichman ${ }^{\mathrm{a},}$ Eugene Serabyn $^{\mathrm{a}}$, Yalan Mao, \\ Pierre M. Echternach $^{\mathrm{a}}$, John T. Trauger ${ }^{\mathrm{a}}$, Kurt M. Liewer ${ }^{\mathrm{a}}$ \\ ${ }^{a}$ Jet Propulsion Laboratory, California Inst. of Technology, 4800 Oak Grove Dr., Pasadena, CA USA \\ 91109; ${ }^{b}$ University of Arizona, Steward Observatory, 933 N. Cherry Ave., Tucson, AZ USA 85721; \\ ${ }^{c}$ Lockheed Martin Advanced Technology Center, 3251 Hanover St., Palo Alto, CA USA 94304
}

\begin{abstract}
The NIRCam instrument on the James Webb Space Telescope will have a Lyot coronagraph for high contrast imaging of extrasolar planets and circumstellar disks at $\lambda=2-5 \mu \mathrm{m}$. Half-tone patterns are used to create graded-transmission image plane masks. These are generated using electron beam lithography and reactive ion etching of a metal layer on an antireflection coated sapphire substrate. We report here on the manufacture and evaluation of the flight occulters.
\end{abstract}

Keywords: James Webb Space Telescope, NIRCam, coronagraph, extrasolar planets

\section{INTRODUCTION}

\subsection{The NIRCam coronagraph}

The NIRCam ${ }^{1}$ instrument on the James Webb Space Telescope (JWST) will provide imaging over $\lambda=1-5 \mu \mathrm{m}$. It will include a Lyot coronagraph for the suppression of diffracted starlight to allow high contrast imaging of exoplanets and circumstellar disks. The coronagraph includes a variety of amplitude masks located at the telescope focus and aperture masks (Lyot stops) located at subsequent images of the telescope pupil. Together these suppress the prominent diffraction pattern of a star created by the segmented JWST primary mirror and other obscurations in the telescope. The performance is expected to be limited by the scattered light created by uncorrected JWST wavefront errors that are budgeted for $131 \mathrm{~nm}$ RMS at the telescope focus ${ }^{2}$.

Details of the NIRCam coronagraph design and performance estimates have been previously published ${ }^{3}$. The fabrication procedures and preliminary tests of prototype occulter samples have also been described ${ }^{4}$. Here we discuss the qualification of the occulters selected for flight.

\subsection{Occulters}

The NIRCam coronagraph utilizes a variety of masks (occulters) at the focus of JWST that block the central portion of a star's point spread function (PSF). An aperture mask (the Lyot stop) is located at a subsequent image of the entrance pupil and blocks the remaining light from the star, allowing faint surrounding objects such as planets or dust disks to be viewed. There are three circular patterns scaled to provide an inner working radius of $6 \lambda / \mathrm{D}$ radians at $\lambda=2.1,3.35$, and $4.3 \mu \mathrm{m}$ (corresponding to 0.4 ", 0.64 ", and 0.82 ", respectively). There are also two wedge-shaped occulters sized from 2 $-6 \lambda / \mathrm{D}$ at $\lambda=2.1 \mu \mathrm{m}$ and $4.6 \mu \mathrm{m}(0.14 "-0.41 "$ and $0.29 "-0.87$ ", respectively).

The occulters are binary half-tone patterns composed of $2 \mu \mathrm{m}-4.3 \mu \mathrm{m}$ wide squares that approximate greyscale transmission masks. Using e-beam lithography, the dots were etched out of a chrome-on-aluminum coating that was deposited on a multilayer, anti-reflection (AR) coating over a sapphire substrate. Also located on the same substrate, under the AR coating and at the edges of the imaging field, are $5 \times 5 \operatorname{arcsec}$ and $2 \times 2$ arcsec squares of nichrome with an optical density of $\sim 3$. These act as neutral density filters to allow unsaturated imaging of a bright star for target acquisition prior to coronagraphic observations.

NIRCam has two identical, mirror-symmetric, redundant modules (A and B), each with a coronagraph, so two flight occulter substrates are needed. The two modules have different pattern layouts to account for vignetting. In addition to the flight substrates, there will be two backups, one for each module, and one additional backup for one of the modules, for a total of five.

Space Telescopes and Instrumentation 2010: Optical, Infrared, and Millimeter Wave, edited by Jacobus M. Oschmann Jr., Mark C. Clampin, Howard A. MacEwen, Proc. of SPIE Vol. $7731,77313 \mathrm{~J} \cdot$ C 2010 SPIE · CCC code: 0277-786X/10/\$18 - doi: 10.1117/12.856488 


\subsection{Lyot stops}

There are two Lyot stops, one for the circular occulters and one for the wedges. They are metallic patterns deposited on optical wedges that are located in the NIRCam filter wheel. At the time of writing, they had just been fabricated and will not be discussed here further.

\section{FLIGHT OCCULTER FABRICATION}

The $66.0 \times 21.7 \times 2.0 \mathrm{~mm}$ sapphire substrates have measured surface roughnesses of $<2.5$ Angstroms RMS. Barr Associates applied coatings in the follow steps:

1. Nickel-chrome neutral density squares (optical density $\approx 3$ ) on the front surface

2. Multilayer antireflection and protective coatings on both sides

3. $\sim 180 \mathrm{~nm}$ thick aluminum layer on the front surface (optical density $>5$ ) on the front side

4. $\sim 20 \mathrm{~nm}$ thick chrome layer on top of the $\mathrm{Al}$ layer to protect the $\mathrm{Al}$ during etching

Witness samples were also coated along with the flight substrates at each stage to provide traceability. The coated substrates were sent to the Micro Devices Lab (MDL) at the Jet Propulsion Laboratory. MDL applied a lithographic resist coating on top of the chrome layer and exposed the regions that were to be opaque (the dots in the patterns) to an electron beam to harden the resist. Both wet and plasma etching were used to remove the metal under the unexposed regions of resist down to the AR coating.

\section{FLIGHT OCCULTER TESTING AND QUALIFICATION}

\subsection{Testing and qualification procedure}

The witness samples were used to verify the wavelength transmission of the AR coating from $\lambda=1.5-5.0 \mu \mathrm{m}$ and the optical density of the ND and Al layers. To prevent possible damage, the flight candidate substrates were untouched after coating at Barr and sent directly to JPL/MDL. At receipt at JPL and prior to processing at MDL the substrates were examined using an optical microscope in a clean room to look for any significant pinholes or scratches in the Al layer that would immediately disqualify the substrate (pinholes $>5 \mu \mathrm{m}$ in diameter near the centers of the to-be-created patterns must be avoided). After etching the substrates were again examined with the clean room microscope in both transmitted and darkfield reflected light to look for pinholes in the ND squares and occulter patterns, anomalous areas of unetched metal (which could be caused by a dirty substrate or impurities in the metal), and any scratches or etching rinse residue. Transmitted light images of the occulter patterns were taken from which the transmission profiles were derived and compared to the specifications.

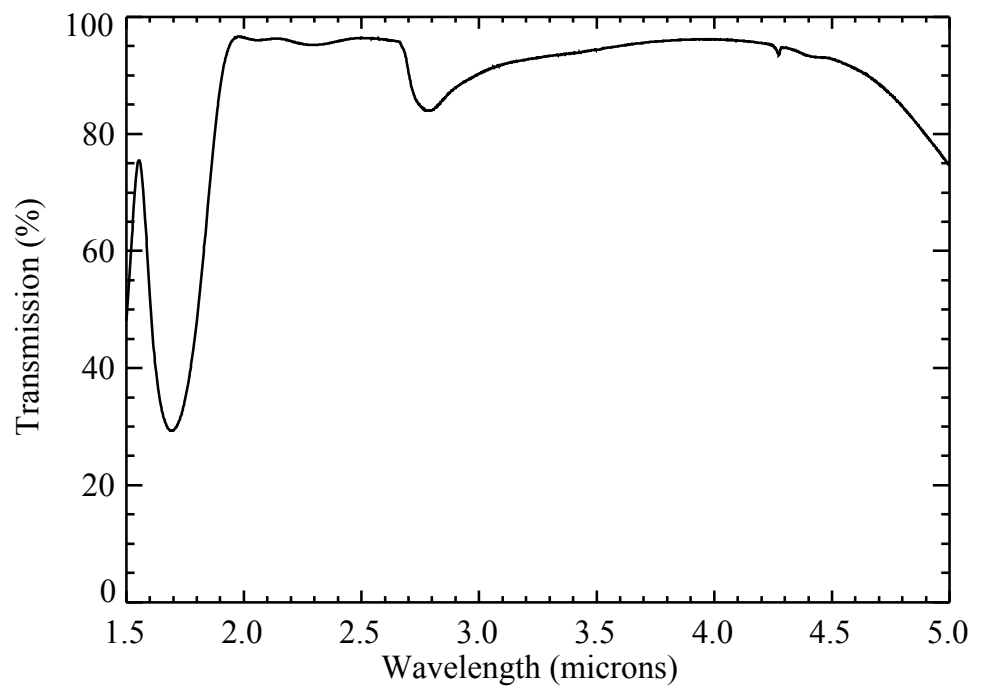

Figure 1. Antireflection coating transmission measured on a witness sample (coating on both sides) at a temperature of $39 \mathrm{~K}$. 


\subsection{Antireflection coating transmission measurements}

The antireflection coating was designed to work over $\lambda=2-5 \mu \mathrm{m}$ and also to provide sufficient transmission at $\lambda=0.633$ $\mu \mathrm{m}$ to allow for alignment using a HeNe laser. The transmission curve was measured at temperatures of $39 \mathrm{~K}$ and $300 \mathrm{~K}$ using witness samples that had just the AR coating (Figure 1). The transmission varies greatly at $\lambda<2 \mu \mathrm{m}$, but because of other issues (chromatic aberration of the pupil image at the Lyot stop due to refractive optics), shorter wavelength coronagraphic observations will not be supported. The measured transmission at $\lambda=0.633 \mu \mathrm{m}$ was $34 \%$, which is acceptable.

\subsection{Neutral density and Al layer optical density measurements}

The optical densities of the neutral density squares and $\mathrm{Al}$ coating were measured at $39 \mathrm{~K}$ and $300 \mathrm{~K}$ over $\lambda=1.5-5$ $\mu \mathrm{m}$. The OD of the ND squares varied between $2.8-3.4$ depending on wavelength. The Al coating was OD $>5$ (OD $>4$ was required).

\subsection{Pre- and post-etching inspections}

The substrates received to date have not had any large pinholes in the Al layer within the regions of the patterns that would preclude continuation to etching (pinholes in other regions are not important since the all of the $\mathrm{Al}$ there would be etched away regardless). The pinholes have largely been below $2 \mu \mathrm{m}$ in size and at low densities. Earlier engineering test samples had larger numbers and sizes of pinholes that were traced to surface contaminants on the AR coating; these were significantly reduced for the flight candidate substrates by a thorough cleaning the coating chamber immediately prior to deposition.

At the time of writing, two flight candidate substrates have been obtained, both with Module A pattern layouts. Additional coated substrates are on order. The two Module A pieces, substrates \#4 (Figure 2) and \#5, have been thoroughly examined and have been deemed flight quality. Transmitted-light microscope images were taken of the occulters (Figure 3) from which mean radial amplitude transmission profiles were derived (Figure 4). The curves matched the desired profiles well. Any errors in the sizes or shapes of the halftone dots would result in deviations of the transmission curves from the desired profiles, but they appear properly sized and formed (Figure 5).

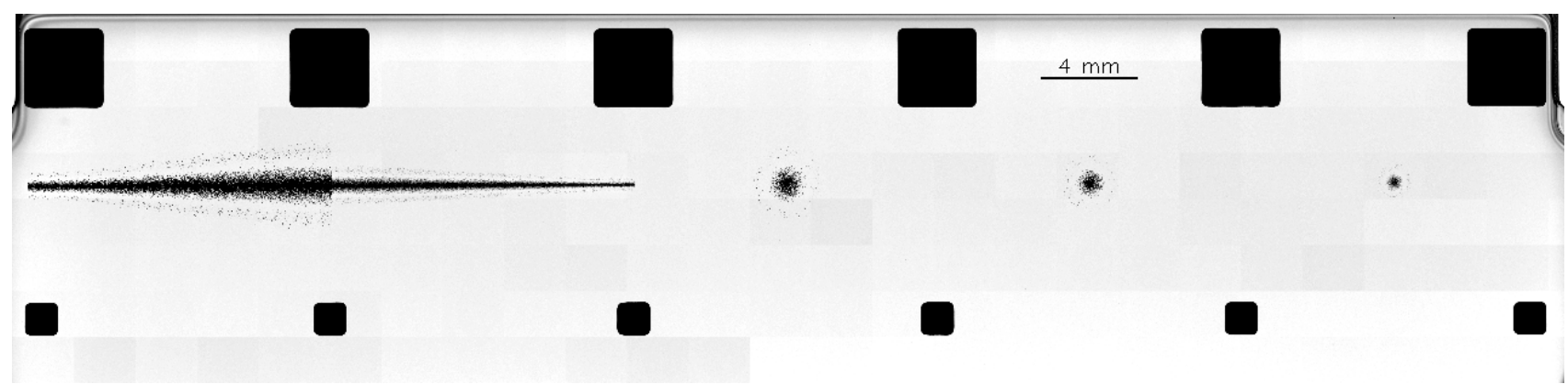

Figure 2. Mosaic of transmitted light microscope images of flight-qualified substrate \#4 (variations in lamp illumination cause the tiling of the background). Along the top and bottom edges are neutral density squares. The wedge on the left provides imaging to within $2 \lambda / \mathrm{D}-6 \lambda / \mathrm{D}$ radians at $\lambda=4.6 \mu \mathrm{m}$ while the smaller one to the right of it is for $\lambda=2.1 \mu \mathrm{m}$. At the center is the $6 \lambda / \mathrm{D}$ occulter scaled for $\lambda=4.3 \mu \mathrm{m}$ and to the right are similar spots for $\lambda=3.35 \mu \mathrm{m}$ and $2.1 \mu \mathrm{m}$. The imaging area, bounded by and including the ND squares, is $60 \mathrm{~mm} \times 12 \mathrm{~mm}(12 \mathrm{~mm}=19 \operatorname{arcsec})$.

Large pinholes in the ND squares could affect the calculation of the centroid of the stellar PSF during target acquisition, and this would lead to an error in positioning the star on the occulter, reducing the contrast performance. Each occulter has two large ND squares nearby, either of which could be used for acquisition if one of them has defects. The squares examined so far have a small number of $<2 \mu \mathrm{m}$ pinholes, which is considered acceptable (the PSF core is $40-100 \mu \mathrm{m}$ wide). There are some $\sim 10 \mu \mathrm{m}$ pinholes along the edges of the large squares, but those are beyond the acquisition regions. Two of the small squares on substrate $\# 5$ contain significant scratches, so the alternative squares would have to 
be used. In addition, there is some residual unetched $\mathrm{Al}$ on substrate \#5, so it will be used as a backup to substrate \#4, the current prime flight piece (pending processing of the other substrates that are on order).

Figure 3. Transmitted light microscope image of the medium-sized occulting spot on substrate \#4.

The image is $1.8 \mathrm{~mm}$ high. This halftone pattern is composed of $3.35 \mu \mathrm{m}$ squares.

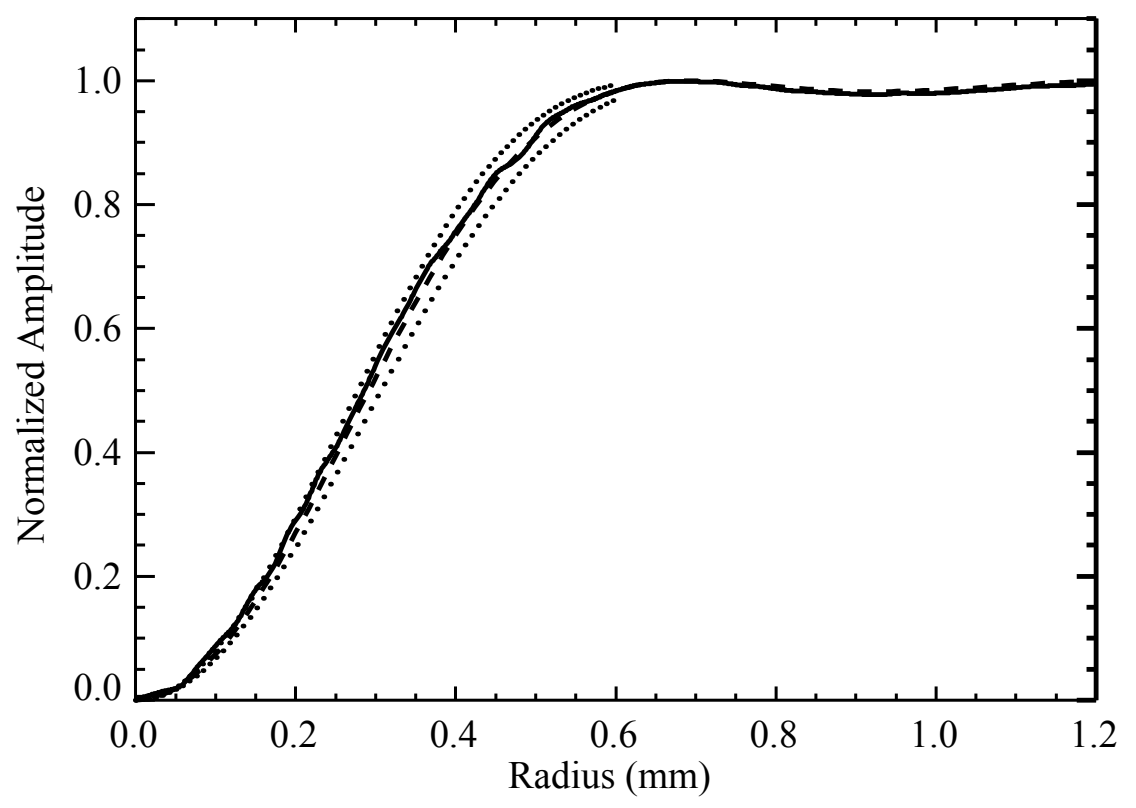

Figure 4. Mean radial profile of medium occulting spot measured from the transmission microscope image shown in Figure 3 (solid line) compared to the desired profile (dashed) and the tolerances (dotted). The finite nature of the dots introduce some noise in the curve, which has been smoothed. 


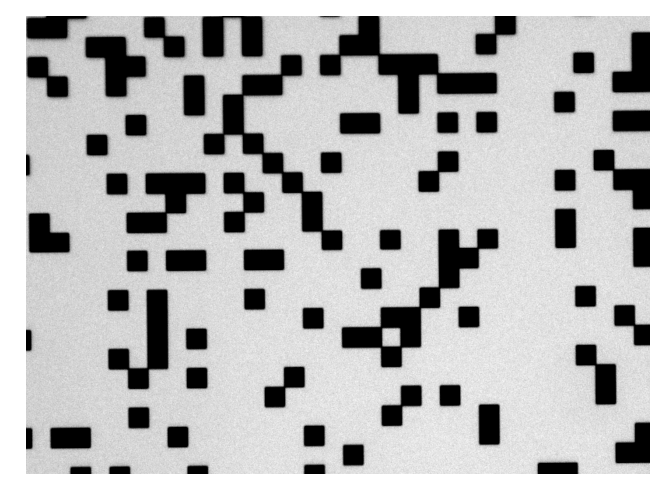

Figure 5. High magnification transmitted light microscope image of a portion of the large occulting spot on substrate \#4. The squares that comprise this halftone pattern are $4.3 \mu \mathrm{m}$ on a side.

Examination of the front and back substrate surfaces was done with a microscope using reflected darkfield illumination. This emphasizes any surface contamination and scratches, including features not seen in transmitted light. Residue from etch rinsing agents was a primary contaminant, though it was significantly reduced by additional rinses (any abrasive cleaning must be avoided since the aluminum is unprotected and easily scratched).

Another substrate, \#3, which is identical to the flight candidates, was used for additional, potentially destructive tests. It was subjected to repeated exposures to helium baths to verify the cryogenic integrity of the substrate and coatings, Tape tests were also done to show that the occulter dots would not flake off during handling or launch. The substrate and coatings survived all of the tests unscathed.

\section{TESTBED EVALUATION OF A FLIGHT-LIKE SUBSTRATE}

Substrate \#3 was evaluated in the InfraRed Coronagraphic Testbed at JPL. This in-air layout is an unobscured system with a circular pupil having the same focal ratio as JWST (f/20). It has an infrared light source feeding a single-mode fiber to produce a point source and a camera that provides imaging out to $\lambda=2.15 \mu \mathrm{m}$, allowing for performance tests of the small spot occulter. Images were taken in the plane of the Lyot stop (Figure 6) after the occulter and in the final image plane using a circular Lyot stop (Figure 7). The pupil plane image provided the most sensitive measure of performance, since the light distribution profile across the bright ring could be compared numerical models. Errors in the occulter patterns would significantly alter the profiles, creating additional rings. The agreement between the observed and model profiles was excellent.
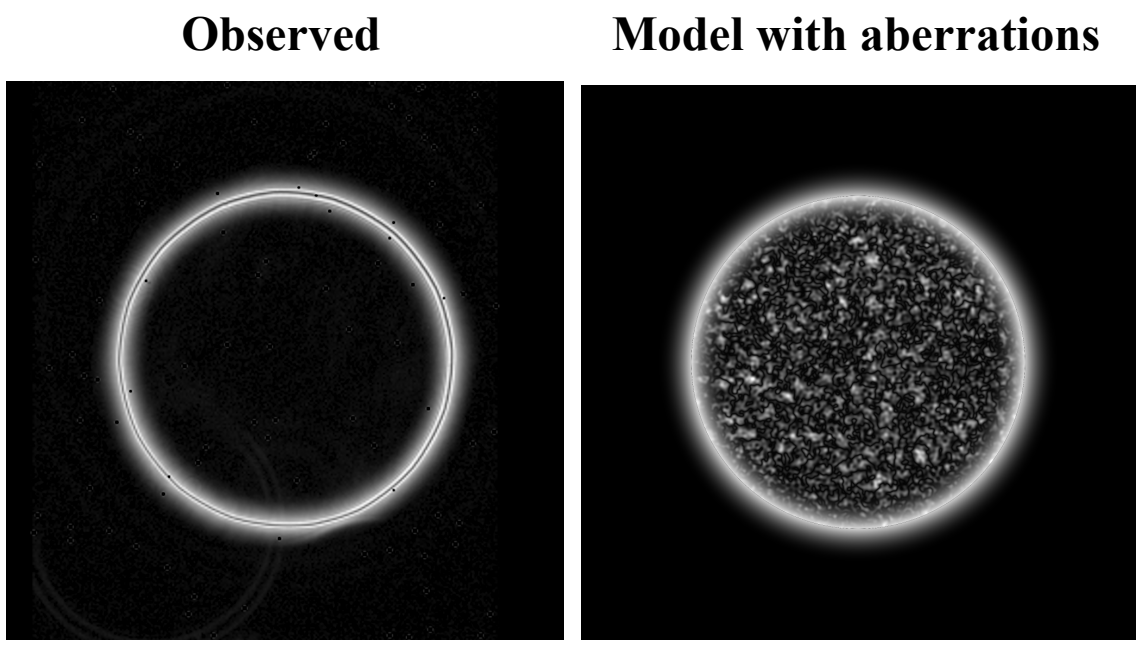

Figure 6. (Left) Observed intensity in the testbed pupil plane after the small occulting spot $(\lambda=2.15$ $\mu \mathrm{m})$. (Right) Model pupil plane intensity for the same configuration with JWST-level aberrations added. 
The pupil image shows that the interior of the pupil is quite dark, down to the scattered light from the testbed aberrations. For the same system, models predict that the occulter blocks $96 \%$ of the light in an unaberrated PSF, and the remainder is rejected at the Lyot stop. Errors in the mask pattern would scatter light into the interior of the pupil, reducing the effectiveness of the Lyot stop and leading to more light in the final image plane. We measured a total rejection factor of about $3 \times 10^{4}$ at final focus, limited largely by the testbed aberrations. This easily meets the desired performance goal of $>50 \times$ rejection. The model pupil image shown in Figure 6 includes JWST-level mid and high spatial frequency wavefront aberrations (55 nm RMS), which are greater than the testbed aberrations. This indicates that the coronagraphic performance will be limited by the JWST wavefront errors and not the occulters.

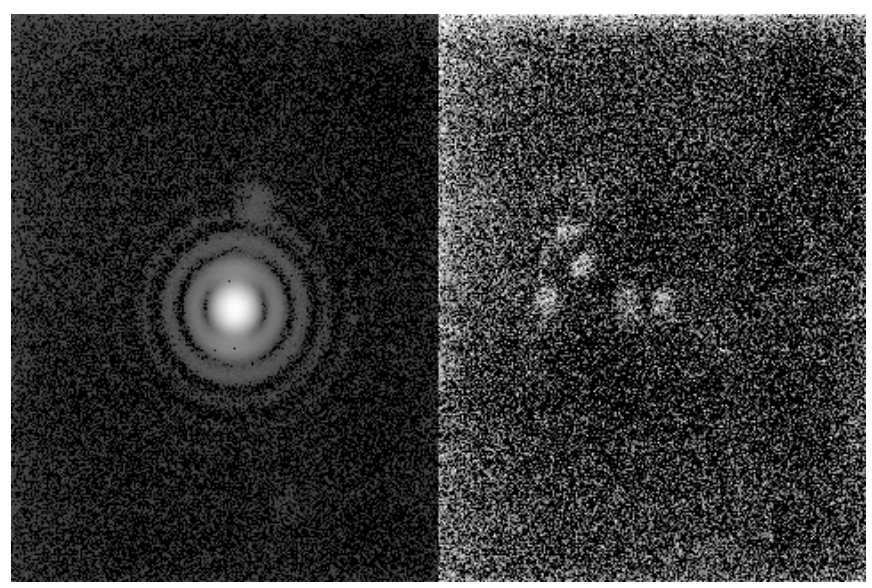

Figure 7. Observed intensity in the testbed final image plane without (left) and with (right) the small occulting spot and a Lyot stop in place $(\lambda=2.15 \mu \mathrm{m})$. The image on the right has been stretched in intensity to demonstrate the suppression of the diffraction pattern.

\section{SUMMARY}

Two flight-quality occulters have been produced for the JWST NIRCam coronagraph. Both of these have pattern layouts for NIRCam Module A. A flight-like occulter was successfully evaluated in a coronagraphic testbed and demonstrated excellent performance. Two Module B occulters and one backup must still be produced.

\section{ACKNOWLEDGEMENTS}

This analysis was performed at the Jet Propulsion Laboratory/Cal. Inst. of Technology and funded by the NIRCam instrument project and science team.

\section{REFERENCES}

[1] Horner, S., Rieke, M., NIRCam team, "The Near Infrared Camera (NIRCam) for the James Webb Space Telescope (JWST)”, Proc. SPIE, 5487, 628 (2004).

[2] Lightsey, P. A., Barto, A. A., Contreras, J., "Optical performance for the James Webb Space Telescope", Proc. SPIE, 5487, 825 (2004).

[3] Krist, J. E., Beichman, C. A., Trauger, J. T., Rieke, M., J., Somerstein, S., Green, J. J., Horner, S. D., Stansberry, J. A., Shi, F., Meyer, M. R., Stapelfeldt, K. R., Roellig, T. L., "Hunting planets and observing disks with the JWST NIRCam coronagraph", Proc. SPIE, 6693, 66390H (2007).

[4] Krist, J. E., et al. "The JWST/NIRCam coronagraph: mask design and fabrication," Proc. SPIE, 7440, 74400W (2009). 